\title{
Empowerment e cura centrata sulla persona: un progetto d'educazione terapeutica dedicato al paziente affetto da ulcere venose. Studio pilota
}

\author{
Katia Feliziani, ${ }^{1}$ Arianna Dichiara, ${ }^{1}$ Giovanna Faccenda, ${ }^{1,2}$ Marco Romanelli, ${ }^{3,4}$ Battistino Paggi ${ }^{5}$ \\ ${ }^{1}$ Distretto Sanitario, ASUR Marche Area Vasta 3, Macerata; ${ }^{2}$ Scienze Infermieristiche, Facoltà di Medicina e Chirurgia, Università \\ Politecnica delle Marche, Ancona; ${ }^{3}$ Dipartimento di Medicina Clinica e Sperimentale, Facoltà di Medicina e Chirurgia, Università degli \\ Studi di Pisa; ${ }^{4}$ Unità Operativa Dermatologia, Dipartimento di Area Medica e Oncologica AOU Pisana; ${ }^{5}$ Consulente per la ricerca clinica \\ e la formazione, Italia
}

\section{RIASSUNTO}

Il progetto ha previsto lo sviluppo, la validazione e la sperimentazione di uno strumento formativo cartaceo dedicato al paziente seguito presso l'ambulatorio infermieristico per ulcere venose dell'arto inferiore. Un infermiere esperto in wound care ha progettato e, in seguito, sottoposto a validazione da parte di un team multiprofessionale un questionario composto da 5 domande concernenti la consapevolezza di malattia, la necessità di essere sottoposti a terapia e la verifica delle conoscenze di alcuni basilari concetti di fisiopatologia. Il questionario è stato somministrato ai pazienti arruolati prima e dopo l'intervento formativo attuato, al fine di testarne l'efficacia (studio pre-post test senza gruppo di controllo). L'analisi dell'efficacia dell'intervento formativo è stata effettuata testando la variazione della media dei punteggi pre (Pre-I) e post intervento (Post-I). Risultati: in base all'analisi statistica effettuata è possibile ritenere l'intervento formativo efficace per il campione selezionato con un buon livello di significatività $(\mathrm{p}<0,001)$ : media dei punteggi Pre-I 6,22 (95\% IC 5,22-7,21) e media Post-I 10,83 (95\% IC 10,59-11,06). In linea con quanto presente in letteratura scientifica, lo studio condotto ha permesso all'infermiere esperto in wound care di sperimentare l'effetto della personalizzazione dell'intervento di cura e dell'empowerment sull'outcome. L'intervento formativo, così

Corrispondenza: Katia Feliziani, Distretto Sanitario, ASUR Marche Area Vasta 3, Largo Belvedere Sanzio 1, 62100 Macerata, Italia. E-mail: katia.feliziani@sanita.marche.it

Key words: Ulcera venosa; empowerment; strumento formativo.

Contributi: KF: ideazione e progettazione dello studio, redazione del manoscritto; AD: analisi e interpretazione dei dati; GF: Supervisione dello studio, coordinamento; RM: supervisione dello studio, coordinamento, approvazione del manoscritto finale; BP: supervisione dello studio, coordinamento, approvazione del manoscritto finale.

Conflitto di interesse: Gli autori non dichiarano nessun conflitto di interessi.

Disponibilità dei dati e materiali: Tutti i dati analizzati in questo studio sono disponibili nel presente articolo.

Approvazione etica e consenso alla partecipazione: Il Direttore di Macrostruttura Territorio, Responsabile del centro nel quale lo studio è stato svolto ha dato l'approvazione alla somministrazione dei questionari.

Consenso alla pubblicazione: I pazienti hanno firmato il loro consenso alla pubblicazione dei dati presenti in questo articolo.

Ricevuto per la pubblicazione: 19 febbraio 2021.

Accettato per la pubblicazione: 17 maggio 2021.

This work is licensed under a Creative Commons Attribution NonCommercial 4.0 License (CC BY-NC 4.0).

${ }^{\circ}$ Copyright: the Author(s), 2021

Licensee PAGEPress, Italy

Italian Journal of Wound Care 2021; 5(2):77

doi:10.4081/ijwc.2021.77 strutturato, pur risultando perfettibile, rappresenta un importante punto di partenza per ulteriori sviluppi futuri.

\section{INTRODUZIONE}

Le ulcere venose degli arti inferiori rappresentano una problematica cronica estremamente invalidante; gravano sul soggetto affetto dal punto di vista clinico, psicologico e sociale. ${ }^{1,2}$

Oltre il 70\% delle lesioni ulcerative degli arti inferiori è di origine venosa. ${ }^{3-5}$

Il rischio di sviluppare insufficienza venosa cronica e lesioni aumenta con l'età. Dato il trend demografico della popolazione, la tendenza sarà quella di avere un numero crescente di pazienti affetti da tale patologia. ${ }^{6-8}$

L'ulcera vascolare venosa degli arti inferiori si caratterizza per una guarigione lenta e per l'alto tasso di ricorrenza; ciò giustifica i costi sanitari e sociali, spesso, significativamente elevati. ${ }^{6,8,9}$

La stima del tasso di recidiva delle ulcere degli arti inferiori varia dal $67 \%$ all' $80 \%$. L'appropriatezza e l'efficacia del percorso di cura, quindi, passano attraverso l'adeguato trattamento delle lesioni, l'intervento tempestivo sullo stile di vita e la correzione dei fattori eziologici sottostanti. ${ }^{10,11}$

La presa in carico del paziente affetto da lesioni vascolari dell'arto inferiore deve avvenire ad opera di un team multidisciplinare, composto da diverse figure professionali, che con le proprie competenze collabora per ottimizzare il percorso di cura. ${ }^{12-16}$ Secondo la letteratura 
presa in esame l'esperto in Wound Care è la figura che meglio si addice al ruolo di supervisore e coordinatore di tutto il team di professionisti. ${ }^{12}$

A giocare un ruolo fondamentale nel percorso di cura dell'ulcere venose degli arti inferiori è l'empowerment del paziente, ovverosia la consapevolezza che il paziente ha di sé e della sua condizione di salute e della sua partecipazione attiva al processo di cura. L'empowerment si concretizza con l'educazione sanitaria e la promozione all'autogestione. ${ }^{17,18}$

L'educazione sanitaria è un processo di apprendimento sistematico, continuo, strutturato e centrato sul paziente.

Deve essere strutturata, organizzata e fornita a tutti i pazienti con una varietà di mezzi. È finalizzata ad aiutare il paziente e il caregiver a comprendere la natura della malattia e la necessità dei trattamenti.

La possibilità per il paziente di avere indicazioni e informazioni scritte sintetiche con un linguaggio pesato sul target e consultabili in qualsiasi momento incoraggiano la compliance e migliorano l'outcome. ${ }^{19-24}$

L'opuscolo cartaceo che contenga informazioni concernenti la patologia della quale si è affetti, norme di condotta e stili di vita si presta bene a rappresentare un potenziale strumento formativo di supporto all'equipe che si occupa della presa in carico del paziente. ${ }^{7,25-28}$

Quindi, partendo da quanto appreso in letteratura si è deciso di ideare e sperimentare un opuscolo formativo cartaceo dedicato al paziente con lesioni vascolari venose degli arti inferiori che accedeva regolarmente all'ambulatorio infermieristico del Distretto di Macerata- Asur Marche AV3, al fine di verificare l'efficacia di questo strumento formativo nel percorso di cura.

\section{EPIDEMIOLOGIA}

Le ulcere degli arti inferiori rappresentano un grave problema clinico e sociale con una prevalenza che oscilla tra lo 0,1 e l' $1 \%$ nella popolazione generale. I dati epidemiologici estratti dalla letteratura internazionale sono spesso discordanti, data la variabilità dei criteri utilizzati per la conduzione degli studi di prevalenza. In Europa la prevalenza attuale della Malattia Venosa Cronica (MVC) a carico degli arti inferiori oscilla tra il 10 e il $50 \%$ nella popolazione adulta maschile e tra il 50 e il $55 \%$ in quella femminile.

L'insufficienza venosa cronica rappresenta la causa principale dello sviluppo delle lesioni ulcerative per una percentuale di casi che oscilla tra il 70 e $1^{\prime} 80 \%{ }^{29}$

In Europa quasi il $70 \%$ dei soggetti affetti afferisce alle classi CEAP C0 e C1, circa il $25 \%$ alle $\mathrm{C} 2$ e $\mathrm{C} 3$ con varici e / o edema; il $5 \%$ dei pazienti può essere stratificato nelle classi da $\mathrm{C} 4$ a C6. ${ }^{30-32}$

Il lungo iter diagnostico-terapeutico, le significative perdite in ore lavorative e le ripercussioni sulla qualità di vita alimentano $i$ costi sanitari causa specifici. ${ }^{33-35}$

La prognosi delle ulcere è poco favorevole tendendo esse a guarire in tempi lunghi e a recidivare con grande facilità. Il 50-75\%, infatti, ripara in 4-6 mesi mentre il $20 \%$ resta attivo a 24 mesi e $1{ }^{\prime} 8 \%$ a 5 anni. ${ }^{36}$

Se si considera la popolazione anziana, il dato di prevalenza risulta pari all' $1,7 \%$ circa, mentre l'incidenza si attesta intorno all' $1,42 \% .{ }^{37}$

La maggior parte dei pazienti viene curata dai servizi territoriali, inoltre circa la metà dei pazienti in carico richiede un trattamento a domicilio. ${ }^{38}$

In Italia il dato di prevalenza delle ulcere degli arti inferiori nella popolazione generale si aggira intorno all' $1 \%$. Circa il $22 \%$ della casistica è costituito da soggetti giovani, ma il numero di pazienti affetti aumenta con l'età. Circa il 3-4\% della popolazione con più di 65 anni manifesta un'ulcera e di questi circa il $75 \%$ è affetto tre o più patologie che possono ostacolarne la guarigione. Nel $75 \%$ dei casi circa le ulcere degli arti inferiori hanno origine venosa; nel $21 \%$ dei casi si manifestano bilateralmente e con una spiccata tendenza alla recidiva entro 5-10 anni. $^{35-38}$

\section{MATERIALI E METODI}

La sperimentazione, autorizzata dal Direttore di Macrostruttura Territorio-Distretto di Macerata e approvata dai pazienti arruolati mediante apposita informativa scritta e modulo di consenso, ha previsto l'ideazione e validazione di uno strumento formativo e di un questionario. Questi sono stati ideati e validati da un team multiprofessionale composto da: psicologa, infermiere esperto in wound care, dermatologo, medico responsabile dell'ambulatorio di ferite difficili, chirurgo vascolare esperto in wound care, medico di medicina generale, fisiatra, medico del distretto di appartenenza dell'ambulatorio infermieristico (promotore dello studio) e grafico.

Lo strumento formativo un opuscolo cartaceo, che si compone di una parte grafica, con immagini stilizzate ed elementari, e di una parte nozionistica, costruita con un linguaggio non tecnico e pensato sul target di destinazione.

Il questionario si compone di cinque domande ed indaga sulla consapevolezza che il paziente ha della sua malattia e dei trattamenti a cui viene sottoposto e verifica le conoscenze di alcuni concetti fisiopatologici di base (Allegato 1).

I questionari, le note informative e i consensi informati sono stati illustrati e consegnati sempre dallo stesso infermiere presso l'ambulatorio delle ferite difficili; nello specifico tale documentazione è stata fornita a ciascun paziente nel momento in cui è stato arruolato nel campione oggetto dello studio. Lo stesso infermiere ha curato anche l'aspetto dell'educazione sanitaria, proposta ai pa- 
zienti già in carico all'ambulatorio infermieristico ed in possesso dei criteri di inclusione previsti dal disegno dello studio. La modalità del colloquio scelta è stata quella di posizionare all'interno di una stanza un tavolo e due sedie una accanto all'altro così da garantire sia la privacy della persona che una comunicazione bidirezionale, potenziando la disponibilità all'ascolto e al confronto.

\section{Disegno dello studio}

Pre-post test senza gruppo di controllo.

\section{Campione in studio}

Soggetti inclusi nello studio: Tutti i pazienti affetti da lesioni venose e miste degli arti inferiori già afferenti all'ambulatorio infermieristico del distretto di Macerata e pazienti seguiti in assistenza domiciliare dal periodo 06/05/2019 al periodo 31/07/2019 che hanno firmato il consenso informato alla partecipazione allo studio, di qualsiasi età, genere, nazionalità e livello di istruzione, senza caregiver.

Soggetti esclusi dallo studio: Pazienti afferenti all'ambulatorio infermieristico del distretto di Macerata e seguiti in assistenza domiciliare, affetti da altra tipologia di lesioni cutanee degli arti inferiori, pazienti con necessità di caregiver e pazienti che non hanno firmato il consenso informato alla partecipazione allo studio.

\section{Analisi statistica}

È stata effettuata un'analisi dell'effetto dell'intervento/strumento formativo sulla variazione dei punteggi ottenuti dai pazienti arruolati nello studio; la significatività statistica è stata testata mediante test $t \mathrm{a}$ una coda per dati appaiati. Per tale elaborazione è stato utilizzato il software XLStat.

\section{RISULTATI}

Il campione di pazienti che rispettano i criteri di inclusione allo studio è composto da 20 pazienti in età compresa tra 42 e 88 anni. Dal campione sono stati eliminati 2 soggetti perché non responders al questionario post intervento formativo.

Il campione in studio è composto da $\mathrm{N}=10$ donne $(55,6 \%)$ e $\mathrm{N}=8$ uomini $(44,4 \%)$. Il $94,5 \%$ dei pazienti $(\mathrm{N}=17)$ è di nazionalità italiana, $\mathrm{N}=1$ paziente è ucraino $(5,5 \%)$. L' $88,9 \%(\mathrm{~N}=16)$ dei pazienti è stato testato in ambito ambulatoriale, mentre per 2 pazienti $(11,1 \%)$ l'infermiere ha eseguito l'intervento formativo e ha somministrato il questionario a domicilio.

Nella Tabella 1 è rappresentata la distribuzione di frequenza delle età per sesso dei pazienti arruolati nello studio.

Nella Figura 1 è rappresentata la distribuzione del livello di istruzione dei pazienti afferenti al campione in studio.

Tabella 1. Distribuzione del campione di pazienti arruolati stratificati per sesso. Età media, deviazione standard, mediana, moda e range.

\begin{tabular}{|c|c|c|c|c|c|}
\hline \multirow{2}{*}{\multicolumn{2}{|c|}{$\mathrm{N}(\%)$}} & \multicolumn{4}{|c|}{ Età } \\
\hline & & Media ( \pm DS) & Mediana & Moda & Range \\
\hline F & $10(55,6 \%)$ & $72,5( \pm 11,8)$ & 74,5 & 81 & $50-88$ \\
\hline M & $8(44,4 \%)$ & $70,37( \pm 14,11)$ & 75 & n.v.* & $42-83$ \\
\hline Totale & $18(100 \%)$ & $71,56(12,56)$ & 74,5 & 81 & $42-88$ \\
\hline
\end{tabular}

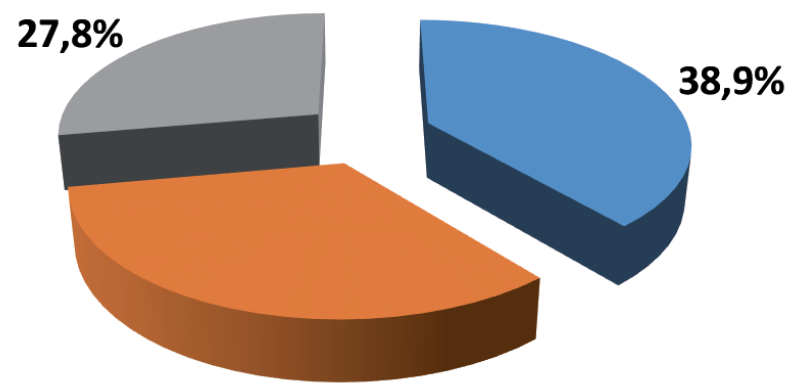

$33,3 \%$

Licenza Elementare

Licenza Media

Diploma Scuola Secondaria di Secondo Grado

Figura 1. Distribuzione del livello di istruzione dei pazienti arruolati al momento dello studio. 
Nella Figura 2 è rappresentata la distribuzione dei punteggi Pre-Intervento (Pre-I) e Post-Intervento formativo (Post-I) nel campione di pazienti considerati.

È stata effettuata un'analisi dell'effetto dell'intervento/strumento formativo sulla variazione dei punteggi ottenuti dai pazienti arruolati nello studio mediante test $t$ a una coda per dati appaiati (Tabella 2).

\section{DISCUSSIONE}

L'analisi condotta ha permesso di evidenziare l'efficacia di un intervento formativo applicato ad un campione selezionato di pazienti seguiti dall'ambulatorio infermie- ristico del Distretto di Macerata - ASUR Marche AV3 nel periodo 6 maggio 2019 - 31 luglio 2019

L'illustrazione del progetto, la somministrazione dei questionari e dell'opuscolo hanno richiesto per paziente circa 40 minuti di cui molto tempo è stato speso per la lettura dell'informativa e per la condivisione del consenso informato.

Dall'analisi condotta è evidente che il genere, l'età e il livello d'istruzione non hanno influito in maniera statisticamente significativa sull'efficacia dell'intervento. Inoltre né l'età, né la scolarità sono risultati discriminanti ai fini dell'interesse e della partecipazione mostrate dal soggetto.

Tabella 2. Punteggi Pre-I e Post-I nel campione di pazienti in esame. Statistica applicata: test $\mathrm{t}$ a una coda per dati appaiati.

\begin{tabular}{lccc}
$\begin{array}{l}\text { Variabili } \\
\text { Sesso }\end{array}$ & Pre-I; media, mediana (IC95\%) & Post-I; media, mediana (IC95\%) & \\
\hline F & 5,$80 ; 1,99(4,56-7,03)$ & 10,$8 ; 0,63(10,41-11,19)$ & 0.003 \\
\hline M & 6,$75 ; 2,38(5,10-8,39)$ & 10,$88 ; 0,35(10,63-11,12)$ & \\
Età & & 11,$00 ; 0,0(0,0-0,0)$ & 0.061 \\
$<70$ & 6,$80 ; 1,64(5,36-8,23)$ & 10,$71 ; 0,75(10,19-11,22)$ & 0.033 \\
\hline $71-79$ & 5,$28 ; 2,13(3,80-6,75)$ & 10,$83 ; 0,40(10,51-11,15)$ & 0.660 \\
\hline 80 & $6,83 ; 2,484,84-8,81)$ & 10,$71 ; 0,76(10,04-11,38)$ & 0.033 \\
\hline Livello di istruzione & & 10,$83 ; 0,40(10,51-11,15)$ & 3.125 \\
\hline Licenza elementare & 5,$28 ; 2,13(3,41-7,14)$ & 11,$0 ; 0,0(0,0-0,0)$ & 2.232 \\
\hline Licenza Media & 6,$83 ; 2,48(4,81-8,81)$ & 10,$83 ; 0,51(10,59-11,06)$
\end{tabular}

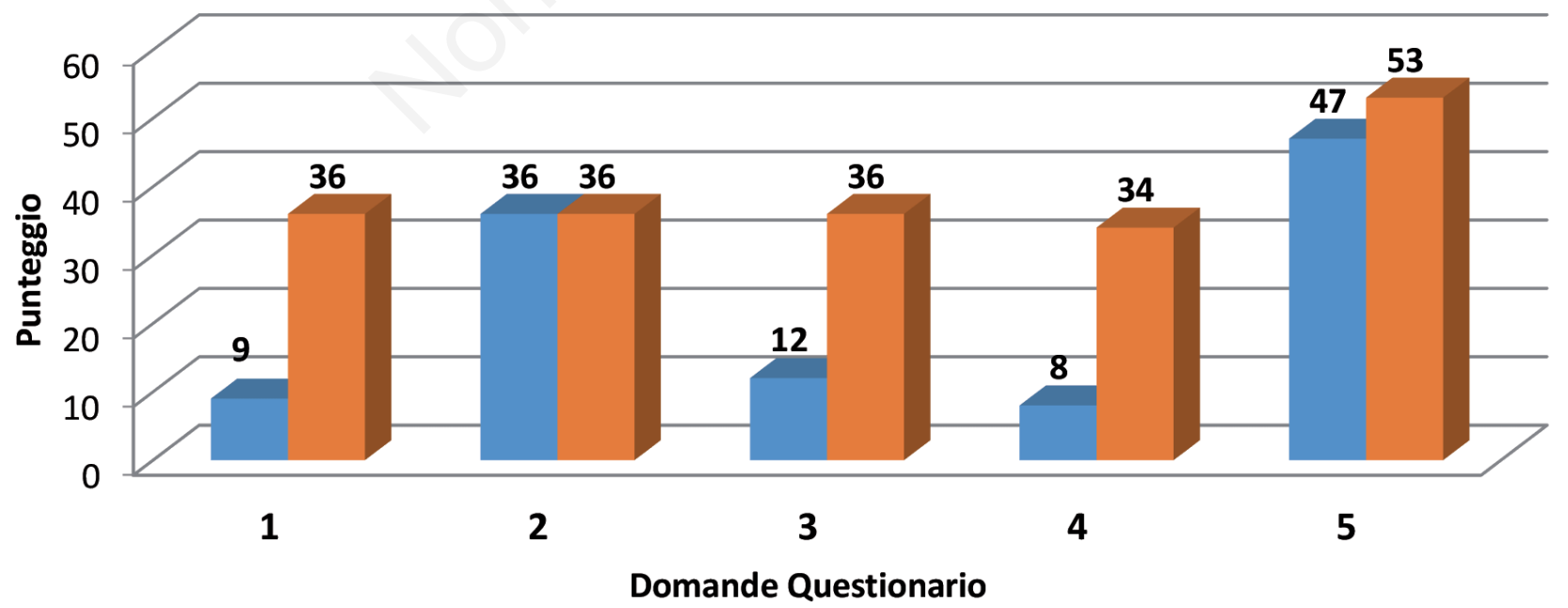

Pre-I Post-I

Figura 2. Distribuzione dei punteggi Pre-I e Post-I ottenuti in seguito a somministrazione del questionario nel campione arruolato. 
Per quanto concerne la verifica della consapevolezza di malattia e la conoscenza di nozioni base di fisiopatologia da parte dei pazienti arruolati, la storia clinica non è stata determinante: pazienti con lesioni che si ripetevano da più di 15 anni non avevano nessuna nozione riguardante il sistema venoso e la patologia in essere.

Dall'elaborazione dei risultati si evidenzia una mancanza quasi completa di concetti elementari di anatomia e fisiologia del sistema venoso e delle cause che conducono all'insufficienza venosa.

Lo studio pilota è stato condizionato da diversi bias.

Il primo limite riguarda l'esiguità della dimensione campionaria determinata dalla ridotta disponibilità temporale in cui si è svolto lo studio. L'utilizzo di un intervallo temporale maggiore avrebbe permesso, infatti, di ottenere un campione maggiormente rappresentativo della popolazione generale.

Il secondo limite è la modalità di somministrazione del questionario in quanto è stato dedicato un unico operatore con formazione specifica in wound care. Qualora la somministrazione dello strumento fosse stata curata da più operatori, ciascuno con diversa esperienza formativa, si sarebbe potuto testare la riproducibilità del test espressa in termini di variabilità intra/inter-operatore.

Il terzo limite ha riguardato la selezione per patologia dei soggetti arruolati; quindi il test statistico è stato utilizzato in un campione estremamente selezionato.

Inoltre non è stato possibile standardizzare il tempo impiegato per la somministrazione dell'opuscolo e del questionario: per alcuni pazienti, infatti, la ridotta collaborazione ha reso più difficoltoso l'intervento allungando i tempi di esecuzione.

Al fine di limitare i missing legati ai non responders, si è scelto di somministrare l'intervento ed effettuare la lettura dell'opuscolo formativo nell'arco della giornata in cui è avvenuto l'accesso ambulatoriale.

Nonostante, nella fase preliminare l'infermiere dedicato alla somministrazione dello strumento abbia avuto modo di testare il feedback del paziente, non è possibile stimare l'efficacia dell'intervento formativo sulla compliance terapeutica di medio-lungo termine (dato in linea con quanto evidenziato in letteratura).

Infine un ultimo limite dello studio è legato alla disomogeneità delle caratteristiche lesione-specifiche; non tutti i pazienti arruolati, infatti, presentavano la stessa storia di malattia: alcuni di essi erano plurilesionati con una storia clinica molto lunga, mentre altri erano al loro primo accesso ambulatoriale.

\section{CONCLUSIONI}

$\mathrm{Al}$ termine dello studio effettuato si può concludere che nel percorso di cura di un paziente affetto da ulcere venose agli arti inferiori gioca un ruolo fondamentale l'empowerment dell'assistito e una attenta e personalizzata educazione sanitaria fatta da un professionista esperto in wound care.

È evidente, anche, come le Raccomandazioni dettate dalle Linee Guida internazionali debbano essere personalizzate e plasmate, per quanto possibile, in base all'esperienza di malattia, alla vita personale, alla condizione familiare del paziente.

Quindi, l'augurio è che questa sperimentazione sia solo l'inizio di altri interessanti studi sull'empowerment del paziente affetto da ulcere venose degli arti inferiori.

\section{BIBLIOGRAFIA}

1. Cavorsi GP. Venous ulcers of the lower extremities: current and newer management techniques. Topics Geriatric Rehab 2000;16:24-34.

2. Charles H. The impact of leg ulcers on patients' quality of life. Prof Nurse 1995; 10:571-4.

3. Morgan PA, Moffatt CJ. Non healing leg ulcers and the nurse-patient relationship. Part 1: the patient's perspective. Int Wound J 2008;5:340-8.

4. Ashton J. Managing leg and foot ulcers: the role of Kerraboot. Br J Community Nurs 2004;9:S26-30.

5. Hampton S. The complexities of heel ulcers. Nurs Stand 2003;17(31): 68-70, 72, 74 passim.

6. Goldman MP, Fronek A. Consensus paper on venous leg ulcer. J Dermatol Surg Oncol 1992;18:592-602.

7. Pickwell KM, Siersma VD, Kars M, et al. Diabetic foot disease: impact of ulcer location on ulcer healing. Diabetes Metab Res Rev 2013;29:377-83.

8. Christensen CR, Yeager AA. Case study: a unique approach to compliance in a patient with venous ulcers. J Vasc Nurs 2001;19:52-4.

9. Moore Z, Cowman S. A systematic review of wound cleansing for pressure ulcers. J Clin Nurs 2008;17: 1963-72.

10. Wounds UK. Two-component compression: Concordance, evidence and clinical use. London: Wounds UK, 2014. Supplement. Available to download from: https://www. wounds-uk.com/resources/details/two-componentcompression-concordance-evidence-and-clinical-use

11. Gilchrist B. Innovations in leg ulcer care. J Wound Care 1998; 7:151-2.

12. Moore Z, Butcher G, Corbett LQ, et al. AAWC, AWMA, EWMA Position Paper: Managing Wounds as a Team. J Wound Care 2014;23:S1-38.

13. Gottrup F, Holstein P, Jørgensen B, Lohmann M, Karlsmar T. A New Concept of a Multidisciplinary Wound Healing Center and a National Expert Function of Wound Healing. Arch Surg 2001;136:765-72.

14. Kelechi TJ, Mueller M, Hankin CS, et al. A randomized, investigator-blinded, controlled pilot study to evaluate the safety and efficacy of a poly-N-acetyl glucosamine-derived membrane material in patients with venous leg ulcers. J Am Academy Dermatol 2012;66: e209-15.

15. Umeh NI, Ajegba B, Buscetta AJ, et al. The psychosocial impact of leg ulcers in patients with sickle cell disease: I 
don't want them to know my little secret. PLoS One 2017;12:e0186270.

16. Armstrong DG, Lavery LA, Harkless LB. Validation of a diabetic wound classification system. The contribution of depth, infection, and ischemia to risk of amputation. Diabetes Care 1998;21:855-9.

17. Van Hecke A, Beeckman D, Grypdonck M, et al. Knowledge deficits and information-seeking behavior in leg ulcer patients: an exploratory qualitative study. J Wound Ostomy Continence Nurs 2013;40:381-7.

18. Edwars LM, Moffatt CJ, Franks PJ. An exploration of patients' understanding of leg ulceration. J Wound Care 2002;11:35-9.

19. Hyland ME, Ley A, Thomson B. Quality of life of leg ulcer patients: questionnaire and preliminary findings. J Wound Care 1994:3:294-8.

20. Audit Commission. What Seems to be the Matter: Communication between hospitals and patients. London; HMSO: 1993.

21. Becker MH, Maiman LA. Sociobehavioral determinants of compliance with health and medical care recommendations. Med Care 1975;13:10-24.

22. Cameron C. Patient compliance: recognition of factors involved and suggestions for promoting compliance with therapeutic regimens. J Adv Nurs 1996;24:244-50.

23. d'Ivernois JF, Gagnayre R, d'Ivernois F, Gagnayre R. Educare il paziente. Un approccio pedagogico, Edizione Italiana a cura di Albano MG, Sasso L. McGraw-Hill: Milano; 2009.

24. Scottish Intercollegiate Guidelines Network. The care of patients with chronic leg ulcer. Sign Publication 1998;26.

25. Goode ML. Giving information on compression to patients with venous leg ulcers. Br J Nurs 2005; 14:1178-9.

26. Clarke Moloney M, Moore A, Adelola OA, et al. Information leaflets for venous leg ulcer patients: are they effective? J Wound Care 2005;14:75-7.

27. Clarke-Moloney M, Keane N, Kavanagh E. Changes in leg ulcer management practice following training in an Irish community setting. J Wound Care 2008;17:116-21.

28. O'Brien JF, Perry IJ, Grace P, Burke PE. Prevalence and aetiology of leg ulcers in Ireland Irish. Irish J Med Sci 2012;169:110-2.

29. Rabe E, Berboth G, Pannier F. Epidemiologie der chronischen Venenkrankheiten [Epidemiology of chronic venous diseases]. Wien Med Wochenschr 2016;166:260-3.

30. Chukwuemeka N, Etufugh T, Phillips J. Venous Ulcers. Clinics Dermatol 2007;25:121-30.

31. Fernandes Abbade LP, Lastória S. Venous ulcer: epidemiology, physiopathology, diagnosis and treatment. Int J Dermatol 2005;44:449-56.

32. Evans R, Ott C, Reddy M. Pressure injury and pressure ulcers. In Griffiths CEM, Barker J, Bleiker T, Chalmers R, Creamer D (eds). Rook's Textbook of Dermatology, 9th Edition. 2016.

33. Carpentier PH, Maricq HR, Biro C, et al. Prevalence, risk factors, and clinical patterns of chronic venous disorders of lower limbs: A population-based study in France. J Vasc Surg 2004;40:650-9.

34. Agus GB, Allegra C, Arpaia G, et al. Linee Guida. Collegio Italiano di Flebologia Revisione 2013. Acta Phlebol 2013;14:1-160.

35. Amato B, Compagna R, Fappiano F, et al Diagnosi differenziale delle anomalie vascolari degli arti inferiori: review. Acta Phlebol 2014;15:95-105.

36. Margolis DJ, Bilker W, Santanna J, Baumgarten M. Venous leg ulcer: incidence and prevalence in the elderly. J Am Acad Dermatol 2002;46:381-6.

37. Marston WA, Carlin RE, Passman MA, et al. Healing rates and cost efficacy of outpatient compression treatment for leg ulcers associated with venous insufficiency. J Vasc Surg 1999;30:491-8.

38. Nebbioso G, Petrella F. Il portale italiano sulle lesioni croniche cutanee: Ulcere arti inferiori. Ulcere venose. 